\title{
A One-pot Access to Diastereoselective Benzo[5,6]oxepino[2,3- c]pyrroles via Formal (5+2)-Annulation of Donor-/Acceptor- Type Aryl Vinyl diazosuccinimide with Ketones Under Silver Catalysis
}

\author{
Debasish Laha, ${ }^{a}$ Kajal B. Meher, ${ }^{a}$ Onkar S. Bankar ${ }^{a}$ and Ramakrishna G. Bhat ${ }^{\text {a* }}$ \\ aDepartment of Chemistry, Indian Institute of Science Education and Research (IISER)-Pune, Dr. Homi Bhabha Road, \\ Pashan, 411008, Pune, Maharashtra, India.
}

\begin{abstract}
A silver triflimide catalyzed one-pot protocol for the diastereoselective synthesis of dihydrobenzoxepines has been developed. Silver triflimide found to be an efficient catalyst for the formal $(5+2)$-cycloaddition to access dihydrobenzoxepines as a single diastereomer using vinyl diazo compounds as 5-C-synthons and ketones. An unmet challenge of utilizing the donor-/acceptor-type vinyl diazosuccinimides as 5-C-synthons under silver-catalysis is presented. This protocol found to be highly regio-, chemo- and diastereoselective, and works well with aliphatic as well as aromatic methyl ketones bearing electron deactivating as well as donating groups to afford dihydrobenzo[5,6]oxepino[2,3-c]pyrroles with a broad substrates scope. The control experiments and time dependent NMR studies revealed the plausible mechanism of this transformation. The protocol also proved to be scalable on the gram scale synthesis.
\end{abstract}

Keywords: Dihydrobenzoxepines; Vinyl diazosuccinimide; Silver catalysis; Annulation; Diastereoselective

\section{Introduction}

The diversity-oriented synthesis (DOS) is a very useful tool for the design and synthesis of new versatile building-blocks of biological importance. ${ }^{[1]}$ The fused polycyclic architecture containing 6-7-5 or 5-7 fused-ring framework such as benzoxepine motif belongs to one of the important classes of oxygenated heterocycles found in pharmaceutical agents ${ }^{[2 a]}$ and numerous natural products ${ }^{[2-d]}$ having diverse biological activities. The stereoselective syntheses of these highly substituted oxygenated heterocycles have immensely been pursued by the scientific community. ${ }^{[3]}$ Directly accessing this scaffold is highly challenging and few approaches have been explored in this regard. ${ }^{[4]}$ It is well known that the transient carbonyl ylide, generated in situ via transition-metal-catalyzed decomposition of diazo compounds can either be paired with suitable trapping agents (or dipolarophiles) or it can be utilized in cyclization (intramolecularly or intermolecularly) to access oxygenated heterocycles. ${ }^{[5-8]} \mathrm{A}$ few methods involving vinyl carbonyl compound have been revealed the preferential formation of dihydrofurans [via (4+1)-annulation] or a mixture of dihydrofurans and dihydrobenzoxepines [via (6+1)-annulation] from specific ylide starting from ester and ketone moiety respectively (Scheme 1A). ${ }^{[8]}$ However, the stereoselective synthesis of either of these scaffolds is quite challenging and it typically needs an elegant ligand-controlled strategy (see Scheme 1B). ${ }^{[9]}$

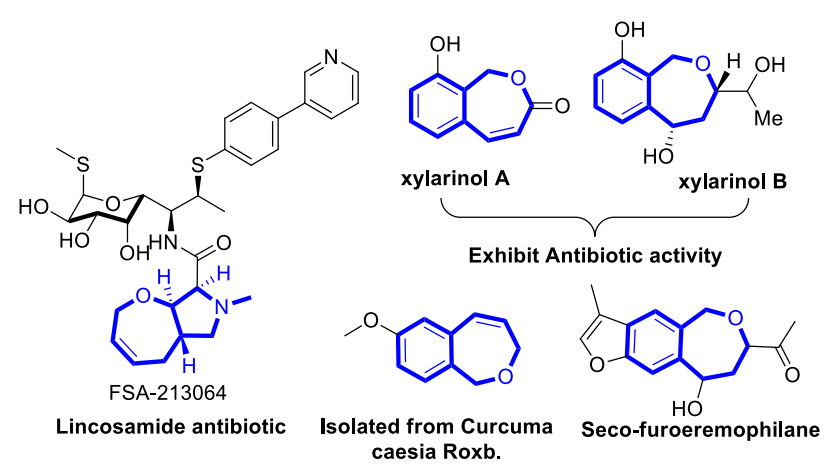

Figure 1. Examples of natural bioactive 1,3dihydrobenzo[c]oxepines and oxepino[2,3-c]pyrrole. 


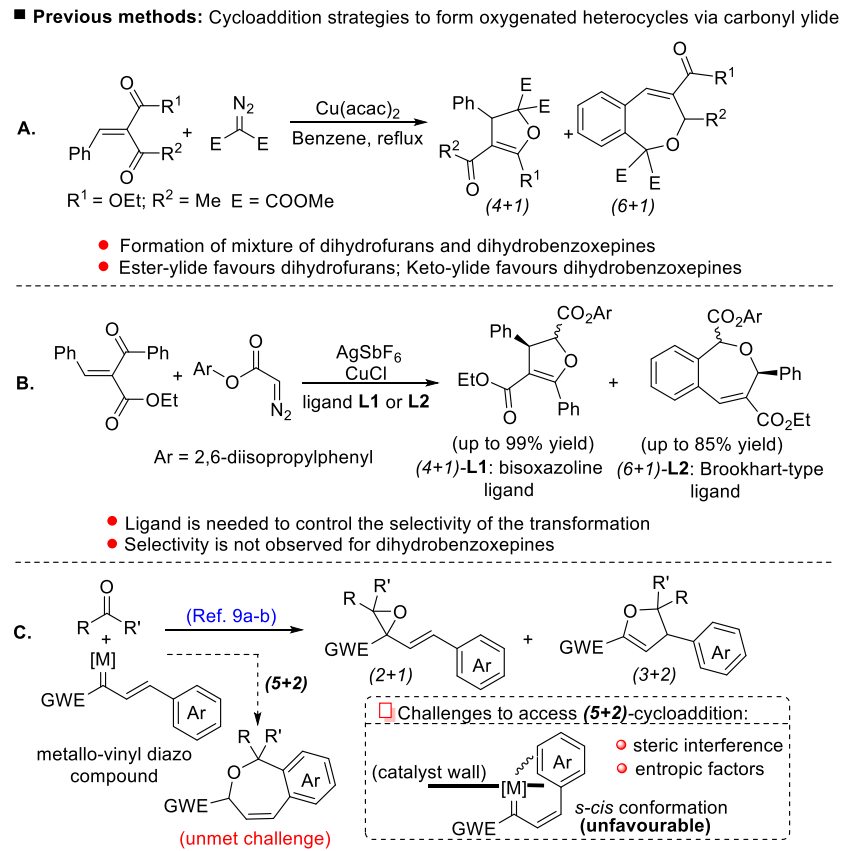

Scheme 1. Access to oxygenated heterocycles via carbonyl ylides.

Though, asymmetric version of some of these reactions have been reported, however, in most of the cases, five-membered heterocycle was obtained exclusively, rather than seven-membered heterocycles. ${ }^{[5],[10]}$ Very recently, Schneider and coworkers reported a protocol for the construction of bicyclic framework: oxa-bridged dibenzooxacines (58 fused rings) via formal (4+3)-cycloaddition reaction involving carbonyl ylide as reactive intermediate. ${ }^{11}$ Besides, the reaction of vinyl diazo compounds with aldehydes usually known to form a mixture of oxiranes [via (2+1)-cycloaddition] and dihydrofurans [via formal $(3+2)$-cycloaddition] through carbonyl ylide involving metal carbene intermediates (Scheme 1C). ${ }^{[12]}$ Ever since these pioneering work, very interestingly, not many investigations have been explored with vinyl diazo compounds. It is important to note that the synthesis of benzoxepine derivatives is quite challenging and moreover cycloannulation via alternative formal $(5+2)$-cycloaddition via carbonyl ylide has not been explored till date to the best of our knowledge; wherein vinyl diazo compounds act as 5$\mathrm{C}$-synthons. The major challenge in this aspect to

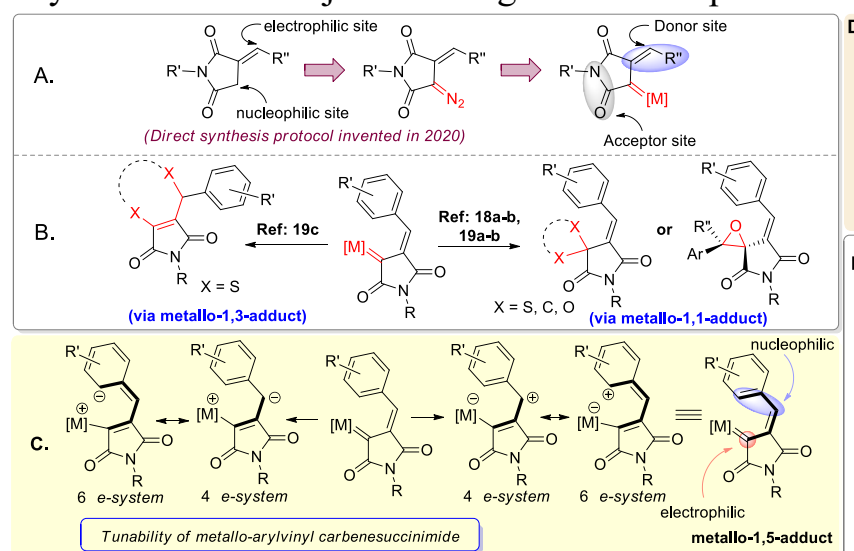

explore the vinyl carbenoid as 5-C synthon is limited due to the intrinsic steric hindrance emanating from $Z$ configuration around the $\mathrm{C}=\mathrm{C}$ bond between benzylidene group and the 'catalyst wall' thus making it unfavorable for the desired transformation (See Scheme 1C inset). ${ }^{[13]}$ Secondly, construction of the seven-membered heterocyclic scaffold such as oxepine via the direct cyclization needs high activation energy mainly owing to the combined effect of entropic factors and the non-bonding interactions in the transition state. ${ }^{[14]}$

The vinyl diazosuccinimide, a useful carbene precursor for the tandem annulation reaction and this can be directly prepared from benzylidene succinimides ${ }^{[15]}$ following our earlier reported procedure and other protocols (Scheme 2A). ${ }^{[16]}$ The metallo-carbenoid of vinyl carbenesuccinimides or vinyl carbenesuccinimides are generated catalytically from stable vinylic diazosuccinimides ( $E$-geometry across $\mathrm{C}=\mathrm{C}$ bond) that exhibit electrophilic character at metal carbene carbon and nucleophilic character at vinylogous carbon (Scheme 2A). These metallo-vinyl carbenesuccinimides have emerged as useful synthons to access metallo-1,1- $-^{[16 a-16 b],[17]}$ or $1,3-^{[17 c]}$ adducts when paired with suitable dipolarophiles or nucleophiles to construct cyclic ring structures involving $\pi$-electrocyclization ${ }^{[16 a],[17 b-d]}$ (Scheme 2B and $2 \mathrm{C}$ ). Looking at these important features and owing to the importance of oxaheterocycles, we envisioned that the metallo-aryl vinyl carbenesuccinimide could be employed for the cycloannulation with carbonyl compounds via $(3+2)$ and/or (5+2)-cycloaddition to access to dihydrofurans and/or dihydrobenzoxepines respectively under suitably optimized reaction conditions (Scheme 2D). Herein, for the first time, we report the silver catalyzed direct diastereoselective synthesis of dihydrobenzoxepine derivatives exclusively by exploring the potential of aryl vinyl diazosuccinimide with ketones under ligand-free condition.

\section{Results and Discussion}

Our previous report had demonstrated the $4 \pi$ electrocyclization strategy via in situ generation of transient carbonyl ylide from metallo-aryl vinyl diazosuccinimide under silver catalysis to access

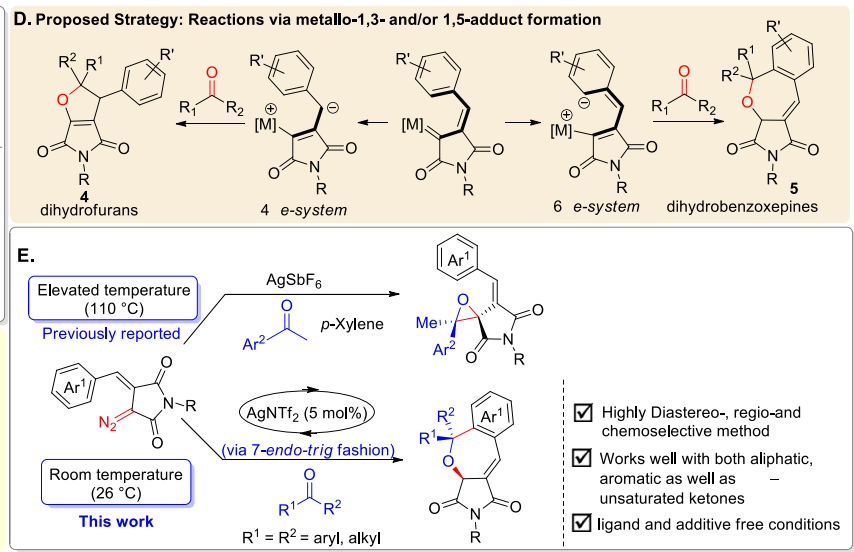

Scheme 2. General characteristics of metallo-aryl vinyl carbenesuccinimide and the current challenges. 
oxiranes at elevated temperature (see Scheme 2E). ${ }^{[6 a]}$ The single crystal X-ray analysis ${ }^{[18]}$ of the phenyl vinyl diazosuccinimide 1a revealed that the benzylidene group is oriented in s-cis conformation with a dihedral angle $(\theta) \sim 34^{\circ}$ (slightly twisted, see SI, page no. S103). Looking at this observation we surmised that even though it is a great challenge, metallo-aryl vinyl diazosuccinimide can be explored for different type of reactivity under suitable conditions wherein aryl group may participate in the reaction as it would be slightly inclined away from the 'catalyst wall' as shown in Table 1. We envisioned that the in situ generated carbonyl ylide (from metallo-aryl vinyl diazosuccinimide) may undergo $6 \pi$-electrocyclization to form dihydrofuran $\mathbf{4}$ or $8 \pi$-electrocyclization followed by rearomatization to afford the dihydrobenzoxepines 5 (see Scheme 2C and D).

Table 1. Selected Optimization Results ${ }^{[\mathrm{a}]}$

$\substack{\text { reduced } \\ \text { sterice } \\ \text { interferences\}) }}$
Entry
face

${ }^{[\mathrm{a}]}$ Reaction conditions: unless otherwise noted, the reactions were carried out with diazo compound 1a (1.0 equiv., 0.2 mmol), 2 a (1.0 equiv., $0.2 \mathrm{mmol}), \operatorname{AgNTf}_{2}(5 \mathrm{~mol} \%)$ and dry $\mathrm{CH}_{2} \mathrm{Cl}_{2}(0.1 \mathrm{M})$ at room temperature $\left(26^{\circ} \mathrm{C}\right)$ under argon atmosphere. ${ }^{[\mathrm{b}]}$ Isolated yields. ${ }^{[\mathrm{c}]} 0{ }^{\circ} \mathrm{C}$; ${ }^{[\mathrm{d}]}-20{ }^{\circ} \mathrm{C}$. ${ }^{[\mathrm{e}]} 60{ }^{\circ} \mathrm{C}$. ${ }^{[\mathrm{f}]}$ Diazo compound $1 \mathrm{a}$ was completely consumed in 2 min at $110^{\circ} \mathrm{C}$. ${ }^{[\mathrm{g}]} \mathrm{dry}_{\mathrm{CH}} \mathrm{Cl}_{2}(0.033 \mathrm{M})$ was used. NR- No reaction. In all cases single diastereomer of 5aa was obtained.

In order to validate our hypothesis, initially we treated (E)-3-benzylidene-4-diazo-1-ethylpyrrolidine2,5-dione 1a with few ketones, aldehydes, amides and esters. Among all the substrates that were screened for the optimization, compound 1a reacted with either acetophenone $\mathbf{2 a}$ or acetone $\mathbf{2 k}$ to afford the desired dihydrobenzoxepines $\mathbf{5 a a}$ or $\mathbf{5 a k}$ as single diastereomer in modest yields respectively (up to $50 \%$, see Appendix-I, SI for details). No trace of product 4 was observed in all of these cases. Encouraged by the initial results, we further sought to optimize the reaction conditions to enhance the yield of diastereomer 5aa. In this regard, we explored the reaction of 1a and 2a using rhodium (Table 1, Entry 1), silver (Table 1, Entries 2-5), copper, iron and gold catalysts in DCM at room temperature (See SI, TableS1 page no. S15-16). Gratifyingly, among all, the reaction of acetophenone $\mathbf{2 a}$ with aryl vinyl diazosuccinimide 1a in presence of silver triflimide $\left(\mathrm{AgNTf}_{2}, 5 \mathrm{~mol} \%\right)$ in dry $\mathrm{CH}_{2} \mathrm{Cl}_{2}$ afforded the corresponding dihydrobenzoxepine 5aa at room temperature (57\% yield, Table 1, Entry 5) along with 3aa as a minor product. The catalytic activity of different silver catalysts was seemed to be dependent on the counter anions. The non-coordinating and low nucleophilic $\left(\mathrm{Tf}_{2} \mathrm{~N}^{-}\right)$counter anion was found to more prominent over others to effect the transformation. We also observed that the reaction did not work in the absence of catalyst for the prolonged reaction time (Table 1, Entry 6). In order to improvise the yield of 5aa and to optimize the reaction conditions further, different solvents, additives, stoichiometric ratio of the substrates, catalyst loading (see SI, Table S2, S4-6) were screened. Later we carefully investigated the other parameters such as temperature (Table 1, Entries 7-11, see SI Table S2 for details) and reactant concentration (Table 1, Entry 10, see SI Table S7). We also observed that in all the cases no trace of compound 4aa was detected during the course of the reaction. Based on the exhaustive screening, we observed that 1a (1 equiv.) and 2a (1 equiv.) in presence of $\operatorname{AgNTf}_{2}(5 \mathrm{~mol} \%)$ as catalyst in dry $\mathrm{CH}_{2} \mathrm{Cl}_{2}(0.033 \mathrm{M})$ at $26{ }^{\circ} \mathrm{C}$ for $2 \mathrm{~h}$ under inert atmosphere proved to be the optimum reaction condition (Table 1, Entry 12).

Having obtained the optimized reaction condition, we planned to extend this protocol for the wider substrate scope. Initially we planned to explore the reactivity of phenyl vinyl diazosuccinimide 1a with different ketones. Different methyl aryl ketones (2a21) reacted with $1 \mathbf{a}$ in presence of silver triflimide (5 mol\%), in dry DCM at room temperature to afford the corresponding 5-aryl-5-methyl substituted dihydrobenzoxepines (5aa-5am) in moderate to good yields (up to $74 \%$, See Table 2). We observed that aryl methyl ketones bearing electron donating groups afforded the corresponding dihydrobenzoxepines (5aa-5ac) in relatively higher yields than that of aryl methyl ketones having electron-deactivating substituents at para- and meta-position of phenyl ring (5ad-5ah). Surprisingly, the desired dihydrobenzoxepine 5ai was not formed as 2,4dichlorophenyl methyl ketone $2 \mathbf{i}$ did not react with 1a under the optimized reaction condition. Further, we explored the reaction of $\mathbf{1 a}$ with electron rich aliphatic methyl ketones including cyclic ketone such as cyclohexanone $\mathbf{2} \mathbf{j}$ to afford the corresponding dihydrobenzoxepine derivatives (5aj-5ak). Even the enone such as methyl styryl ketone reacted with $1 \mathrm{a}$ to give the desired product 5 am in modest yield (50\%, Table 2). 
Later, we planned to explore formal $(5+2)-$ cycloaddition for a range of aryl-substituted and $\mathrm{N}$ substituted aryl vinyl diazosuccinimides with different ketones. In this regard, we treated different $N$ substituted benzylidene diazo compounds (1b-1e) with acetophenone $\mathbf{2 a}$ under optimized reaction condition to afford the corresponding desired products (5ba-5ea) (see Table 2). We observed that among all the $N$-substituted benzylidene diazo compounds screened, $N$-ethyl substituted benzylidene diazo

Table 2. Substrates Scope for the Formal $(5+2)$ cycloaddition reaction $^{[a]}$

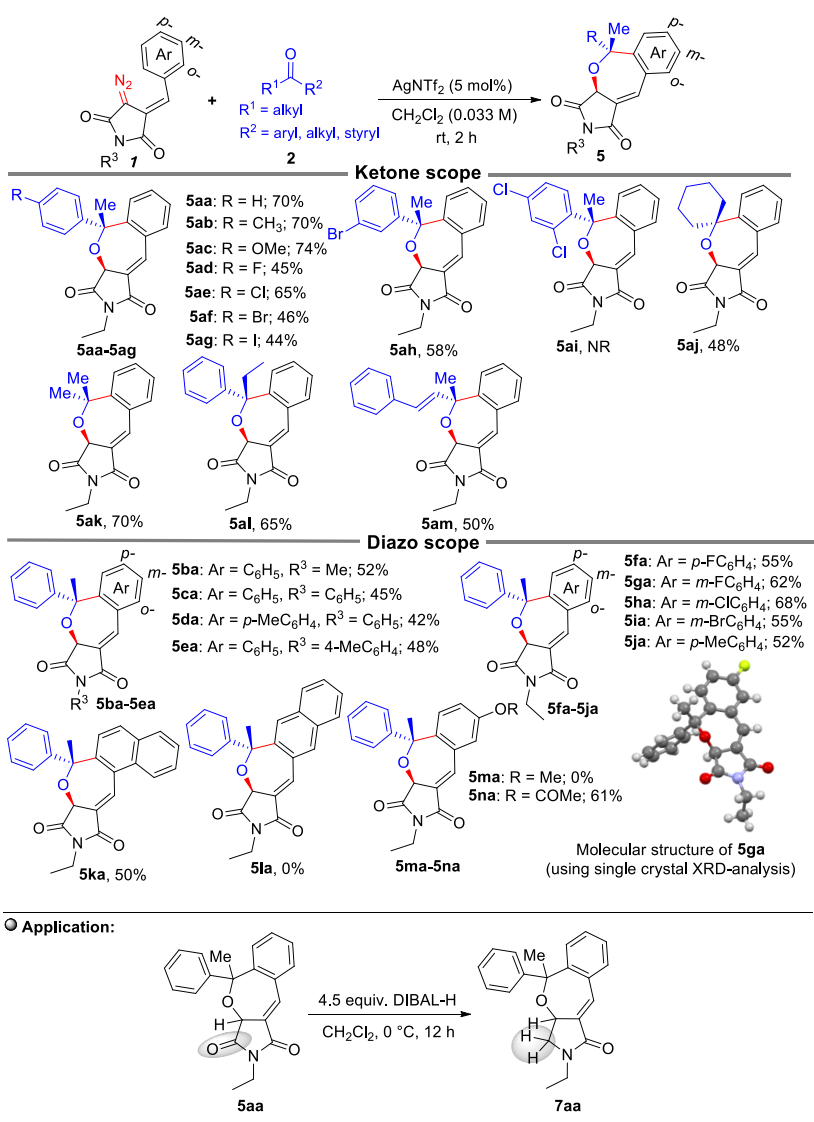

[a]Reaction conditions: unless otherwise noted, the reactions were carried out with diazo compound 1a (1.0 equiv., 0.2 $\mathrm{mmol}), 2$ (1.0 equiv., $0.2 \mathrm{mmol}), \operatorname{AgNTf}_{2}(5 \mathrm{~mol} \%)$ and dry $\mathrm{CH}_{2} \mathrm{Cl}_{2}(0.033 \mathrm{M})$ at room temperature $\left(26^{\circ} \mathrm{C}\right)$ under argon atmosphere over $2 \mathrm{~h}$. In all cases single diastereomer was obtained.

compound 1a proved to be optimum substrate by affording the desired product $\mathbf{5 a a}$ in relatively higher yield. In view of this, we planned to explore the reactivity of different $N$-ethyl substituted vinyl diazo compounds (1f-1n) bearing different substituents with electron-rich and -deficient/-deactivated groups at different positions of phenyl ring of the vinyl diazo succinimide. The reaction of acetophenone $\mathbf{2 a}$ with $N$ ethyl substituted vinyl diazo compounds (1f-1k) under the optimized reaction conditions afforded the target products $(\mathbf{5 f a - 5} \mathbf{k a})$ in moderate to good yields at room temperature in a very short time (up to $68 \%, 2 \mathrm{~h}$ ). It is Interestingly, we obtained the desired product 5aa in $64 \%$ yield after $5 \mathrm{~h}$ (Scheme 3c). interesting that the
$N$-ethyl substituted vinyl diazo compound derived from $\alpha$-napthal (1k) reacted smoothly, while $\beta$-napthal derived derivative (1I) did not react probably due to electronic and steric reasons. We observed that the vinyl diazo compounds bearing electron deactivating groups $(-\mathrm{F},-\mathrm{Cl},-\mathrm{Br})$ at different positions of phenyl ring, afforded the target products in relatively higher yields than that of electron rich system $(\mathbf{1 b - 1 d}, \mathbf{1 j})$. Unfortunately, the $N$-ethyl substituted vinyl diazo compounds having methoxy group (1m) at meta position found to be not reactive. We reasoned that probably due to the presence of electron donating group (-OMe), the carbenoid carbon would be relatively less electrophilic for desirable reaction. In order to validate this, we replaced the methoxy group with the electron deactivating acetyloxy group at the same position (meta, 1n) and treated with acetophenone $\mathbf{2 a}$ under the optimized reaction condition. Gratifyingly, we obtained the corresponding desired product 5na in moderate yield $(61 \%$, Table 2). It is interesting to note that relatively sensitive benzylidene scaffold containing $p$-methyl group at phenyl ring $(\mathbf{5} \mathbf{d a}, \mathbf{5 j a})$ tolerated the optimized reaction conditions. Later, in order to generalize the practicality of the protocol, we explored the reaction of 1a and 2a on gram scale quantity and further as an application, the transformation of 5aa was carried to obtain the pyrrolidinone derivative 7aa (see Table 2 or Appendix-II, SI). All the dihydrobenzoxepines were obtained as single diastereomer. We obtained the molecular structure of 5ga using single crystal X-ray analysis $^{[18]}$ and the structures of all the other compounds were deduced based on analogy.

In order to have an insight into the reaction mechanism, we performed few control experiments systematically. To verify whether or not the reaction undergoes via carbonyl ylide (int-II or III, Scheme 3) or epoxide (int-IV, Scheme 3), we initially prepared the corresponding epoxide ${ }^{[18]}$ (int-IV or 3aa) using the reported procedure. ${ }^{[16 a]}$ This epoxide 3aa under the optimized reaction conditions did not afford the desired product 5aa even in trace and the unreacted epoxide 3aa was recovered (Scheme 3a). Likewise, we observed that reaction did not afford the desired product 5aa when carried out in absence of catalyst at room temperature (Scheme 3b). These results confirm that epoxide 3aa (int-IV) is not the intermediate for the desired transformation at room temperature. The int-II (four different conformational forms of metalbound carbonyl ylides in one inset, see Figure 3) and int-III (four different conformational forms of the corresponding metal-unbound carbonyl ylides) might be generating from the reaction of aryl vinyl diazosuccinimide under silver catalysis, presented here. It is reported by Huisgen that the epoxide can undergo electrocyclic ring opening reaction upon heating (absence of any catalyst). This report confirms about the in situ formation of carbonyl ylide at the room temperature reaction condition. ${ }^{[19 a-b]}$ In this regard, in order to verify the possible formation of intermediate (int-II or III), we further treated compound 3aa (epoxide) with $\operatorname{AgNTf}_{2}(5 \mathrm{~mol} \%)$ at an 
elevated temperature $\left(110{ }^{\circ} \mathrm{C}\right.$, Scheme 3c). Interestingly we obtained the desired product 5aa in $64 \%$ yield after $5 \mathrm{~h}$ (Scheme $3 \mathrm{c}$ ).

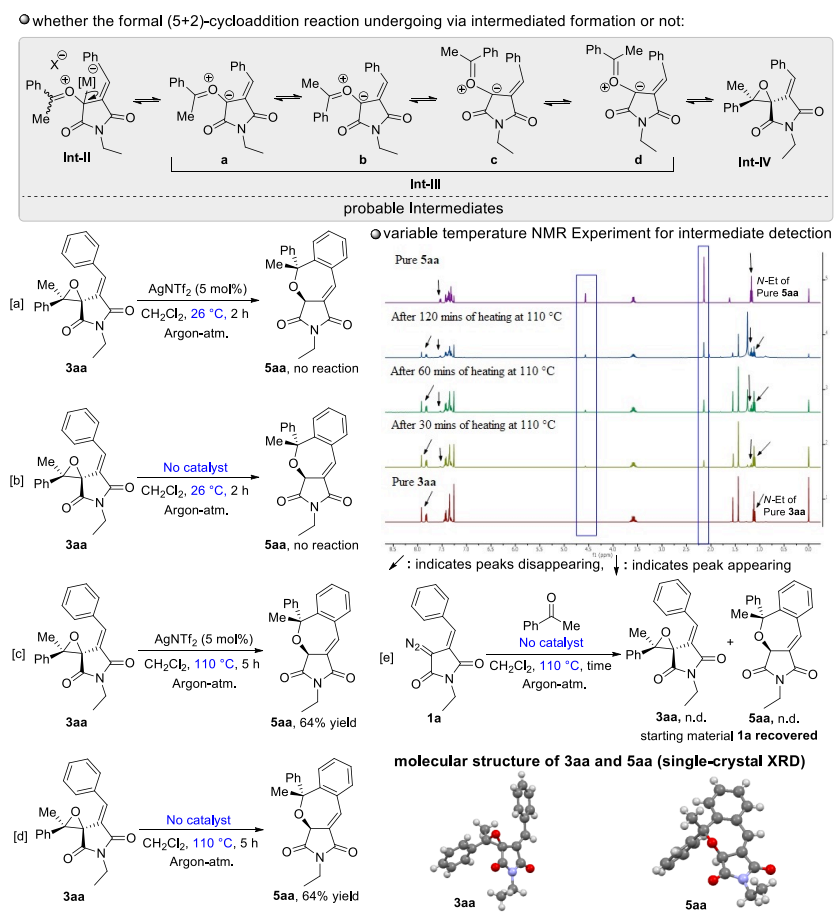

Scheme 3. Control Experiments for Intermediate Detection.

To understand the possible role of silver catalyst as well as carbonyl ylide, we performed the reaction in the absence of $\operatorname{AgNTf}_{2}$ at elevated temperature $\left(110^{\circ} \mathrm{C}\right.$, Scheme 3d). To our surprise we obtained compound $\mathbf{5 a a}^{[18]}$ in almost similar yield (Scheme $3 \mathrm{~d}$, for more details, see SI]. Considering Huisgen's ${ }^{[19 a-b]}$ and Eberbach's reports ${ }^{[19 c]}$ on the electrocyclic ringopening equilibria of oxirane to carbonyl ylide and different conformational forms of carbonyl ylide, we surmise that at an elevated temperature, epoxide 3aa would open up to form metal-unbound carbonyl ylide (see int-IIId, Scheme 3). This result was further supported by the time-dependent ${ }^{1} \mathrm{H}-\mathrm{NMR}$ experiment wherein signals for the epoxide 3aa were disappearing over a period of time at an elevated temperature to form 5aa (Scheme 3, for more details, see SI page no. S34-35). We also strongly believe that even at room temperature the reaction would undergo via the same intermediate (int-IIId) to afford 5aa. Apart from that an attempt to trap the carbonyl ylide intermediate (intIII) with different dienophiles ${ }^{[8 \mathrm{~d}]}$ were unsuccessful. We observed that none of dienophiles reacted with the epoxide (int-IV) either at $26^{\circ} \mathrm{C}$ or at $110^{\circ} \mathrm{C}$. In order to understand the role of catalyst, we carried out the reaction of 1a and acetophenone $\mathbf{2 a}$ in the absence of catalyst at $110^{\circ} \mathrm{C}$ for prolonged reaction time (Scheme $3 \mathrm{e})$. Interestingly, we did not obtain either the desire product 5aa or the epoxide 3aa even in trace thus supporting the role of catalyst in the desired transformation (Scheme 3e). Earlier also we had observed that the reaction at room temperature did not work in the absence of catalyst and the starting material 1a was recovered $\left(26{ }^{\circ} \mathrm{C}\right.$, see Table 1, Entry
6). These two results indicate that probably a reactive silver carbenoid (int-I, see Scheme 4) species might be forming in situ that eventually may be forming carbonyl ylide (int-IIId) via the corresponding counter anion stabilized metal-bound carbonyl ylide (int-IId) both at room temperature as well as at higher temperature. These combined results clearly depicted that the epoxide (int-IV) may not be the favourable intermediate and probably the desired product 5aa might be forming via carbonyl ylide (int-IIId) at room temperature. These control experiments suggest that among the three plausible intermediates, the carbonyl ylide (int-IIId) is the plausible intermediate for the formation of dihydrobenzoxepine 5aa products. Moreover, a metal-unbound (free-ylide) carbonyl ylide intermediate, generating in situ, is proposed to be operative here based on the control experimental observations (for details, see SI page no. S33-35). We surmise relatively better coordinating and nucleophilic counter anion $\mathrm{Tf}_{2} \mathrm{~N}^{-}$might be acting as a better promoter in facilitating the formation of int-IIId faster and easily at room temperature than that of $\mathrm{SbF}_{6}{ }^{-}$ anion. ${ }^{[20]}$ We also noted that the anticipated product 4aa has not been detected during the transformation. This might be the cause of steric hindrance exerted by the benzylidene group (phenyl ring tilted towards the carbonyl moiety) on vinyl succinimide, occurring in the transition state or in the carbonyl intermediate (intIIId)

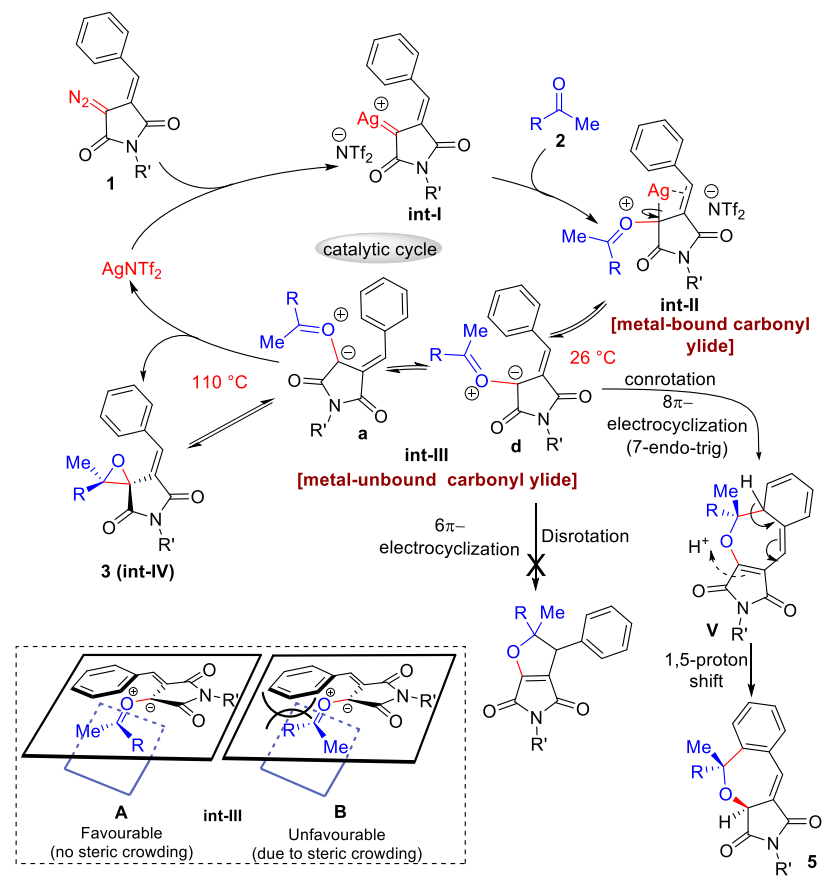

Scheme 4. Plausible Mechanism for the (5+2)-Annulation.

Based on the experimental observations and preceding literatures, ${ }^{[19 b-c, 21]}$ the plausible mechanism has been proposed (See Scheme 4). The vinyl diazosuccinimide 1 under the reaction conditions generates the silver carbenoid (int-I) by the extrusion of dinitrogen. This intermediate (int-I) further reacts with the ketone 2 (approaches from sterically less hindered site; see Scheme 4, inset) to form metal 
unbound carbenoid (int-IIId) via the corresponding counter anion stabilized metal bound carbonyl ylide (int-IId). This intermediate would further undergo $8 \pi$ electrocyclization (7-endo-trig) to species $\mathbf{V}$ which after successive $(1,5)$-proton abstraction afford the corresponding desired oxepine 5. At higher temperature int-IV might be in equilibrium with the int-IIId, can also form the desired product 5aa.

\section{Conclusions}

In summary, we have demonstrated the formal (5+2)-cycloaddition route to access the dihydrobenzoxepines in one-pot using vinyl diazosuccinimide as 5-C-synthons and ketones very effectively for the first time. The silver triflimide proved to be an efficient catalyst for this transformation and the protocol found to be highly regio-, chemo- and diastereoselective. The method proved to be general as it worked well with aliphatic as well as aromatic methyl ketone bearing electron deactivating as well as donating groups to afford the benzo[5,6]oxepino[2,3-c]pyrroles as single diastereomer with a broad substrates scope. The control experiments and time-dependent NMR studies gave an insight into the plausible reaction mechanism of this transformation. The protocol proved to be scalable on gram scale synthesis.

\section{Experimental Section}

\section{General procedure for the synthesis of dihydrobenzo[5,6]oxepino[2,3-c]pyrrole-1,3(2H)-dione (5):}

An oven-dried $25 \mathrm{~mL}$ two necked round bottom (RB) flask equipped with a magnetic stirrer bar was charged with $\mathrm{AgNTf}_{2}$ (3.9 mg, $5 \mathrm{~mol} \%$ ) under argon-atmosphere and the $\mathrm{RB}$ flask was covered up with aluminium foil. Then dry $\mathrm{CH}_{2} \mathrm{Cl}_{2}(5 \mathrm{~mL})$ and acetophenone $2 \mathrm{a}(23.6 \mu \mathrm{L}, 1.0$ equiv., $0.2 \mathrm{mmol}$ ) were added subsequently and the mixture was stirred at room temperature under for a minute. After that, vinyl diazosuccinimide 1 a (48.3 $\mathrm{mg}, 1.0$ equiv., $0.2 \mathrm{mmol}$ ) in $\mathrm{CH}_{2} \mathrm{Cl}_{2}(1 \mathrm{~mL})$ was added to the reaction mixture dropwise over $2 \mathrm{~h}$ and the stirring was continued for $10 \mathrm{~min}$ more for full consumption of diazo compound. The progress of the reaction was monitored by TLC. After completion of the reaction, the solvent was removed under reduced pressure. The crude product obtained was purified by column chromatography (petroleum ether/EtOAc 92:8) over silica gel to furnish the desired product 5aa as white solid (47 mg, 70\% yield). The slight excess amount of acetophenone $\mathbf{2} \mathbf{a}$ was recovered during the purification.

\section{Acknowledgements}

R. G. B. thanks the Science and Engineering Research Board, Department of Science and Technology (SERB-DST), New Delhi, Government of India (File no:CRG/2019/005753) for the generous research grant. D. L., K. B. M. and O. S. B. thank the UGC, New Delhi, Government of India for providing fellowship. The authors thank IISER Pune for the financial assistance. We acknowledge Dr. Rahul Maity and Prakash K. Warghude of IISER Pune for helping in for obtaining the crystal data.

\section{References}

[1] a) M. D. Burke, E. M. Berger, S. L. Schreiber, Science 2003, 302, 613-618; b) M. D. Burke, S. L. Schreiber, Angew. Chem., Int. Ed. 2004, 43, 46-58.

[2] a) A. G. Myers, M. J. Mithceltree, K. J. Silvestre, From PCT Int. Appl. (2009), WO 2019032936 A1 20190214; b) I. K. Lee, Y. W. Jang, Y. S. Kim, S. H. Yu, K. J. Lee, S. M. Park, B. T. Oh, J. C. Chae, B. S. Yun, J. Antibiot. 2009, 62, 163-165; c) F. Bohlmann, G. Fritz, Tetrahedron 1982, 38, 1431; d) A. Ghosh, A. Bandyopadhyay, P. Ghosh, Journal of Scientific and Innovative Research 2013, 2, 795-801.

[3] a) D. Basavaiah, D. S. Sharada, A. Veerendhar, Tetrahedron Lett. 2004, 45, 3081-3083; b) B. Das, A. Majhi, J. Banerjee, N. Chowdhury, H. Holla, K. Harakishore, U. S. Murty, Chem. Pharm. Bull. 2006, 54, 403-405; c) G. Islas- González, J. Benet-Buchholz, M. A. Maestro, A. Riera, M. A. Pericàs, J. Org. Chem. 2006, 71, 1537-1544; d) A. V. Fernndez, C. G. Yebra, J. A. Varela, M. A. Esteruelas, S. Carlos, Angew. Chem. 2010 122, 4374-4377; e) J. Francuz, B. Srećo, M. Popsavin, G. Bene-deković, V. Divjaković, V. Kojić, G. Bogdanović, A. Kapor, V. Popsavin, Tetrahedron Lett. 2012, 53, 1819; f) S. J. Gharpure, S. R. B. Reddy, Eur. J. Org. Chem. 2013, 2981-2984; g) A. G. K. Reddy, J. Krishna, G. Satyanarayana, Tetrahedron 2013, 69, 10098-10107; h) A. A. More, C. V. Ramana, Chem. Asian. J. 2014, 9, 1557-1562.

[4] a) H. Chen, Y. He, L. Zhou, Org. Chem. Front. 2018, 5, 3240-3244; b) M.-Y. Chang, Y.-T. Hsiao, K.-H. Lai, J. Org. Chem. 2018, 83, 14110-14119.

[5] S. Son, G. C. Fu, J. Am. Chem. Soc. 2007, 129, 1046.

[6] For reviews, see: a) M. P. Doyle in Comprehensive Organometallic Chemistry II, Vol. 12 (Ed.: L. S. Hegedus), Pergamon, New York, 1995, chap. 5.2; b) A. Padwa, M. D. Weigarten, Chem. Rev. 1996, 96, 223; c) G. Mehta, S. Muthusamy, Tetrahedron 2002, 58, 9477; d) M. C. McMills, D. Wright in Synthetic Application of 1,3-Dipolar Cycloaddition Chemistry Toward Heterocycles and Natural Products (Eds.: A. Padwa, W. H. Pearson), Wiley, New York, 2002, chap. 4.

[7] a) H. Suga, A. Kakehi, S. Ito, K. Inoue, H. Ishida, T. Ibata, Org. Lett. 2000, 2, 3145; b) H. Suga, A. Kakehi, S. Ito, K. Inoue, H. Ishida, T. Ibata, Bull. Chem. Soc. Jpn. 2001, 74, 1115.

[8] For selected examples, see: a) S. Torssell, M. Kienle, P. Somfai, Angew. Chem. 2005, 117, 3156; Angew. Chem. Int. Ed. 2005, 44, 3096; b) V. Nair, S. Mathai, S. C. Mathew, N. P. Rath, Tetrahedron 2005, 61, 2849; c) V. V. Diev, R. R. Kostikov, R. Gleiter, A. P. Molchanov, J. Org. Chem. 2006, 71, 4066; d) A. DeAngelis, M. T. Taylor, J. M. Fox, J. Am. Chem. Soc. 2009, 131, 1101; e) O. Anaç, Ö. Sezer, Ö. Candan, F. Ş. Güngör, M. ŞerifCansever, Tetrahedron Lett. 2008, 49, 1062-1065.

[9] J.-L. Zhou, Y. Liang, C. Deng, H. Zhou, Z. Wang, X.-L. Sun, J.-C. Zheng, Z.-X. Yu, Y. Tang, Angew. Chem. 2011, 123, 8020-8024. 
[10] J.-L. Zhou, L.-J. Wang, H. Xu, X.-L. Sun, Y. Tang, ACS Catal. 2013, 3, 685-688.

[11] A. Suneja, H. J. Loui, C. Schneider, Angew. Chem. Int. Ed. 2020, 59, 5536-5540.

[12] a) M. Hamaguchi, H. Matsubara, T. Nagai, J. Org. Chem. 2001, 66, 5395-5404. b) H. M. L. Davies, J. DeMeese, Tetrahedron Lett. 2001, 42, 6803-6805.

[13] Y. Lian, H. M. L. Davies, Org. Lett. 2010, 12, 924927.

[14] a) A. Parenty, X. Moreau, J.-M. Campagne, Chem. Rev. 2006, 106, 911-939.

[15] a) X.-Y. Zhang, Y. You, Z.-H. Wang, J.-Q. Zhao, W.C. Yuan, Chem. Commun. 2020, 56, 13449; b) Z. Gan, M. Zhi, R. Han, E.-Q. Li, Z. Duan, F. Mathey, Org. Lett. 2019, 21, 2782; c) B.-L. Zhao, D.-M. Du, Chem. Commun. 2016, 52, 6162.

[16] a) D. Laha, R. G. Bhat, Asian J. Org. Chem. 2020, 9, 918-921; b) E. Chupakhin, M. Gecht, A. Ivanov, G. Kantin, D. Dar'in, M. Krasavin, Synthesis 2021, 53, 1292-1300; c) E. G. Chupakhin, G. P. Kantin, D. V. Dar'in, M. Krasavin, Mendeleev Commun. 2021, 31, 36-38.

[17] a) Y. Nakano, M. Hamaguchi, T. Nagai, J. Org. Chem. 1989, 54, 5912-5919; b) D. Dar'in, G. Kantin, O.
Bakulina, A. Inyutina, E. Chupakhin, M. Krasavin, $J$. Org. Chem. 2020, 85, 15586-15599; c) M. Hamaguchi, T. Misumi, T. Oshima, Tetrahedron Lett. 1998, 39, 7113-7116; d) A. Inyutina, D. Dar'in, G. Kantina, M. Krasavin, Org. Biomol. Chem. 2021, 19, 5068-5071.

[18] CCDC 2105271 (compound 1a), 2105766 (compound 5ga), 2105262 (compound 3aa), and 2105272 (Compound 5aa) contain the supplementary crystallographic data. These data can be obtained free of charge by The Cam-bridge Crystallographic Data Centre.

[19] a) R. Huisgen, Angew. Chem., Int. Ed. Engl. 1977, 16, 572-585; b) P. de March, R. Huisgen, J. Am. Chem. Soc. 1982, 104, 4952. c) W. Eberbach, U. Trostrnann, Chem. Ber. 1985, 118, 4035-4058.

[20] It is believed that tight ion pair can form between silver cation and counter anion $\left(\mathrm{Tf}_{2} \mathrm{~N}^{-}\right)$as it is relatively more confined with closer proximity to metal center than that of $\mathrm{SbF}_{6}{ }^{-}$. [Also, the order of coordinating tendency of these two anions is $\mathrm{SbF}_{6}^{-}<\mathrm{Tf}_{2} \mathrm{~N}^{-}$.

[21] G. Zecchi, Synthesis 1991, 3, 181-188. 
Table of Contents Graphic

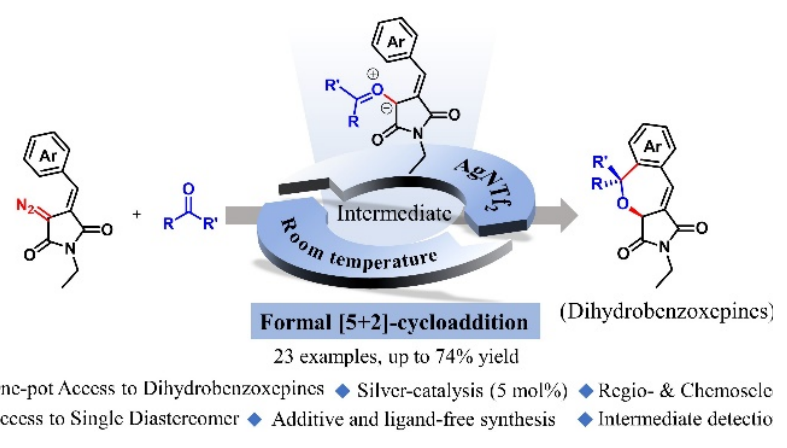

